Article

\title{
Tri- and Tetrahyperbolic Isofrequency Topologies Complete Classification of Bianisotropic Materials
}

\author{
Maxim Durach *, Robert Williamson, Morgan Laballe and Thomas Mulkey \\ Department of Physics and Astronomy, Georgia Southern University, Statesboro, GA 30460, USA; \\ rw05157@georgiasouthern.edu (R.W.); morgan_h_laballe@georgiasouthern.edu (M.L.); \\ tm06381@georgiasouthern.edu (T.M.) \\ * Correspondence: mdurach@georgiasouthern.edu
}

Received: 5 December 2019; Accepted: 14 January 2020; Published: 21 January 2020

\begin{abstract}
We describe novel topological phases of isofrequency k-space surfaces in bianisotropic optical materials - tri- and tetrahyperbolic materials-which are induced by the introduction of chirality. This completes the classification of isofrequency topologies for bianisotropic materials, as we showed that all optical materials belong to one of the following topological classes-tetra-, tri-, bi-, mono-, or nonhyperbolic. We showed that phase transitions between these classes occur in the $\mathrm{k}$-space directions with zero group velocity at high k-vectors. This classification is based on the sets of high-k polaritons (HKPs), supported by materials. We obtained the equation describing these sets and characterized the longitudinal polarization-impedance of HKPs.
\end{abstract}

Keywords: bianisotropic materials; hyperbolic metamaterials; photonics

\section{Introduction}

Hyperbolic topologies have sparked the imaginations of science fiction prosaists for almost a century [1]. In turn, the topology of isofrequency k-surfaces in photonic materials now fascinates the members of the optics community. The known topologies include bounded k-surfaces, such as spheres or ellipsoids, and unbounded k-surfaces-single- and double-leaf hyperboloids [2-4], and recently discovered bihyperboloids [5]. As can be seen from this list of k-surface topologies, the main difference between them and therefore the key to their classification is the propagation or absence of high-k modes in the corresponding materials (Figure 1). The high-k modes, which are the short-wavelength solutions of macroscopic Maxwell's equations in hyperbolic metamaterials, are of primary interest in photonics, and have already found applications for optical imaging in nanoscopic resolution using hyperlenses, emission control due to diverging optical density of high-k states, and emission-directivity control [6].

With the development of nanofabrication, a variety of bianisotropic and chiral metamaterials have been realized, which includes a combination of split-ring resonators, helixes, gammadions, and other metallic shapes in unit cells [6]. In this letter we theoretically predict novel isofrequency topology phases, tri- and tetrahyperbolic materials, and obtain an equation that describes the k-space directions in which the high-k modes exist in terms of the 36 material parameters of an arbitrary bianisotropic material. Furthermore, we employ a theorem from Zeuthen (1873) [7] to show that our prediction of the tri- and tetrahyperbolic topological phases completes the classification of bianisotropic materials, meaning that an optical material belongs to one of the following classes-tetra-, tri-, bi-, mono-, or nonhyperbolic. The novel tetra- and trihyperbolic phases which we predict here are induced via the introduction of chirality. Chirality-induced modification of topology in the energy-momentum space was previously studied for monohyperbolic materials [8]. Here we discuss the topology of isofrequency surfaces in k-space. 


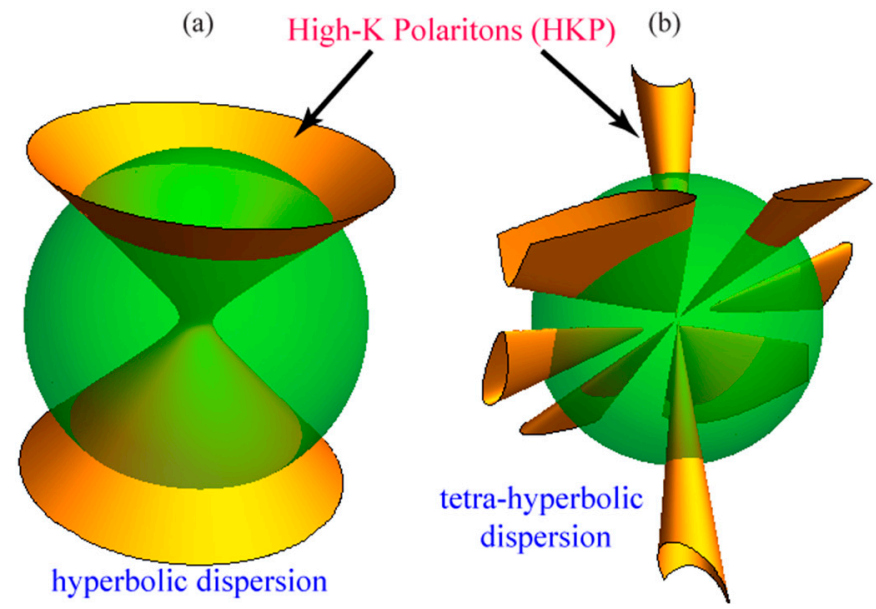

Figure 1. Isofrequency k-surface in k-space with high-k polaritons (HKPs) in a conventional monohyperbolic material (a) and a tetra-hyperbolic material (b) introduced in this paper and described in detail in the discussion below.

We show that in all high-k modes, both electric and magnetic fields are polarized longitudinally. In this sense, these modes are similar [9] to the bulk plasmon polaritons, bulk magnon polaritons, or longitudinal optical phonon polaritons, except that the high-k modes are short-wavelength. Therefore, we shall refer to these modes as high-k polaritons (HKPs). For these HKPs, we explicitly express the ratio between the longitudinal electric and magnetic field, i.e., longitudinal impedance $Z_{l}$, in term of the 36 bianisotropic material parameters. To accomplish this, we introduce the index of refraction operator $\hat{N}$. We obtain the direction-dependent refraction indices explicitly in arbitrary bianisotropic material.

\section{Theory of Isofrequency Surfaces in Bianisotropic Materials}

Consider bianisotropic materials with $6 \times 6$ effective parameters matrix $\hat{M}=\left[\begin{array}{cc}\hat{\epsilon} & \hat{X} \\ \hat{Y} & \hat{\mu}\end{array}\right]$, such that the constitutive relations are given by $\left(\begin{array}{c}\boldsymbol{D} \\ \boldsymbol{B}\end{array}\right)=\hat{M}\left(\begin{array}{c}\boldsymbol{E} \\ \boldsymbol{H}\end{array}\right)$. It is known that the isofrequency surface of the most generic bianisotropic material with arbitrary material parameters $\hat{M}$ is a quartic surface in k-space given by [10].

$$
f\left(k, k_{0}\right)=\sum_{i+j+l+m=4}\left[\alpha_{i j l m} k_{x}^{i} k_{y}^{j} k_{z}^{l} k_{0}^{m}\right]=0 .
$$

As was demonstrated in Ref. [10], the coefficients $\alpha_{i j l m}$ follow from the solution of Maxwell's equation. The topological asymptotic skeleton of the isofrequency surfaces (Equation (1)) can be found in the high-k limit $k \gg k_{0}$. The high-k states are those tending to the conical k-surfaces given by

$$
h(\boldsymbol{k})=f\left(k \rightarrow \infty, k_{0}\right)=\sum_{i+j+l=4}\left[\alpha_{i j l 0} k_{x}^{i} k_{y}^{j} k_{z}^{l}\right]=0
$$

Below we establish the detailed form and properties of the function $h(k)$ in terms of the material parameters $\hat{M}$ and the topological properties of the skeleton asymptotic surfaces of Equation (2). 
We start by considering Maxwell's equations for the amplitudes $\hat{\Gamma}=\left(\begin{array}{c}E \\ H\end{array}\right)$ of the plane waves with wave vectors $k$ and frequencies $\omega=c k_{0}$ written as

$$
k \hat{Q} \hat{\Gamma}=k_{0} \hat{M} \hat{\Gamma}, \hat{Q}=\left[\begin{array}{cc}
\hat{0} & \hat{R} \\
-\hat{R} & \hat{0}
\end{array}\right], \hat{R}=\left(\begin{array}{ccc}
0 & -k_{z} & k_{y} \\
k_{z} & 0 & -k_{x} \\
-k_{y} & k_{x} & 0
\end{array}\right)
$$

Consider a wave propagating in the direction $\hat{k}=k / k=\left(q_{x}, q_{y}, q_{z}\right)$. Let us use the transformation $\hat{T}$ :

$$
\hat{T}=\left(\begin{array}{ccc}
q_{z} q_{x} / q_{\rho} & q_{z} q_{y} / q_{\rho} & -q_{\rho} \\
-q_{y} / q_{\rho} & q_{x} / q_{\rho} & 0 \\
q_{x} & q_{y} & q_{z}
\end{array}\right)=\left(\begin{array}{c}
q_{1} \\
\boldsymbol{q}_{2} \\
\boldsymbol{q}_{3}=\hat{\boldsymbol{k}}
\end{array}\right)
$$

where $q_{\rho}=\sqrt{q_{x}^{2}+q_{y}^{2}}$ and vectors $q_{i}$ are the rows of matrix $\hat{T}$. Note that $\hat{T}=\left(\hat{T}^{T}\right)^{-1}$ and is a proper rotation $\operatorname{det} \hat{T}=1$.

Let us apply transformation $\hat{T}$ to Maxwell's Equation (Equation (3))

$$
\begin{gathered}
k \widetilde{Q \Gamma}=k_{0} \widetilde{M \Gamma}, \widetilde{\Gamma}=\hat{T}_{6} \hat{\Gamma}, \widetilde{Q}=\hat{T}_{6} \hat{Q} \hat{T}_{6}^{-1}=\left[\begin{array}{cc}
\hat{0} & \widetilde{R} \\
-\widetilde{R} & \hat{0}
\end{array}\right] \\
\widetilde{R}=\left(\begin{array}{ccc}
0 & -1 & 0 \\
1 & 0 & 0 \\
0 & 0 & 0
\end{array}\right), \widetilde{M}=\hat{T}_{6} \hat{M} \hat{T}_{6}^{-1}=\left[\begin{array}{cc}
\widetilde{\epsilon} & \widetilde{X} \\
\widetilde{Y} & \widetilde{\mu}
\end{array}\right], \hat{T}_{6}=\left[\begin{array}{cc}
\hat{T} & 0 \\
0 & \hat{T}
\end{array}\right]
\end{gathered}
$$

According to Equation (5) the transformed matrices $\widetilde{a}=\widetilde{\epsilon}, \widetilde{\mu}, \widetilde{X}, \widetilde{Y}$ can be expressed in terms of the original $\hat{a}=\hat{\epsilon}, \hat{\mu}, \hat{X}, \hat{Y}$ as

$$
\widetilde{a}_{i j}=\boldsymbol{q}_{i} \cdot \hat{a} \cdot \boldsymbol{q}_{j}^{T}
$$

The zeroes in the bottom row of matrix $\widetilde{R}$ are due to the conditions $\boldsymbol{k} \cdot \boldsymbol{D}=0$ and $\boldsymbol{k} \cdot \boldsymbol{B}=0$. Thus, the longitudinal components of fields $E_{z}$ and $H_{z}$ can be decoupled from the transverse field components in the transformed coordinates as

$$
\left(\begin{array}{c}
E_{z} \\
H_{z}
\end{array}\right)=-\left(\begin{array}{cc}
\widetilde{\epsilon}_{33} & \widetilde{X}_{33} \\
\widetilde{Y}_{33} & \widetilde{\mu}_{33}
\end{array}\right)^{-1}\left(\begin{array}{cccc}
\widetilde{\epsilon}_{31} & \widetilde{\epsilon}_{32} & \widetilde{X}_{31} & \widetilde{X}_{32} \\
\widetilde{Y}_{31} & \widetilde{Y}_{32} & \widetilde{\mu}_{31} & \widetilde{\mu}_{32}
\end{array}\right)\left(\begin{array}{c}
E_{x} \\
E_{y} \\
H_{x} \\
H_{y}
\end{array}\right),
$$

while the transverse components satisfy the system of equations

$$
\begin{gathered}
k\left(\begin{array}{cccc}
0 & 0 & 0 & -1 \\
0 & 0 & 1 & 0 \\
0 & 1 & 0 & 0 \\
-1 & 0 & 0 & 0
\end{array}\right)\left(\begin{array}{c}
E_{x} \\
E_{y} \\
H_{x} \\
H_{y}
\end{array}\right)=k_{0}\left(\begin{array}{cccc}
\bar{\epsilon}_{11} & \bar{\epsilon}_{12} & \bar{X}_{11} & \bar{X}_{12} \\
\bar{\epsilon}_{21} & \bar{\epsilon}_{22} & \bar{X}_{21} & \bar{X}_{22} \\
\bar{Y}_{11} & \bar{Y}_{12} & \bar{\mu}_{11} & \bar{\mu}_{12} \\
\bar{Y}_{21} & \bar{Y}_{22} & \bar{\mu}_{21} & \bar{\mu}_{22}
\end{array}\right)\left(\begin{array}{c}
E_{x} \\
E_{y} \\
H_{x} \\
H_{y}
\end{array}\right)=k_{0} \bar{m}\left(\begin{array}{c}
E_{x} \\
E_{y} \\
H_{x} \\
H_{y}
\end{array}\right) \\
\bar{m}=\left(\begin{array}{cccc}
\widetilde{\epsilon}_{11} & \widetilde{\epsilon}_{12} & \widetilde{X}_{11} & \widetilde{X}_{12} \\
\widetilde{\epsilon}_{21} & \widetilde{\epsilon}_{22} & \widetilde{X}_{21} & \widetilde{X}_{22} \\
\widetilde{Y}_{11} & \widetilde{Y}_{12} & \widetilde{\mu}_{11} & \widetilde{\mu}_{12} \\
\widetilde{Y}_{21} & \widetilde{Y}_{22} & \widetilde{\mu}_{21} & \widetilde{\mu}_{22}
\end{array}\right)-\left(\begin{array}{cc}
\widetilde{\epsilon}_{13} & \widetilde{X}_{13} \\
\widetilde{\epsilon}_{23} & \widetilde{X}_{23} \\
\widetilde{Y}_{13} & \widetilde{\mu}_{13} \\
\widetilde{Y}_{23} & \widetilde{\mu}_{23}
\end{array}\right)\left(\begin{array}{lll}
\widetilde{\epsilon}_{33} & \widetilde{X}_{33} \\
\widetilde{Y}_{33} & \widetilde{\mu}_{33}
\end{array}\right)^{-1}\left(\begin{array}{cccc}
\widetilde{\epsilon}_{31} & \widetilde{\epsilon}_{32} & \widetilde{X}_{31} & \widetilde{X}_{32} \\
\widetilde{Y}_{31} & \widetilde{Y}_{32} & \widetilde{\mu}_{31} & \widetilde{\mu}_{32}
\end{array}\right) .
\end{gathered}
$$


We rearrange this and introduce the index of refraction operator $\hat{N}$ :

$$
\hat{N}\left(\begin{array}{c}
E_{x} \\
H_{y} \\
H_{x} \\
E_{y}
\end{array}\right)=n\left(\begin{array}{c}
E_{x} \\
H_{y} \\
H_{x} \\
E_{y}
\end{array}\right), \hat{N}=\left(\begin{array}{cccc}
-\bar{Y}_{21} & -\bar{\mu}_{22} & -\bar{\mu}_{21} & -\bar{Y}_{22} \\
-\bar{\epsilon}_{11} & -\bar{X}_{12} & -\bar{X}_{11} & -\bar{\epsilon}_{12} \\
\bar{\epsilon}_{21} & \bar{X}_{22} & \bar{X}_{21} & \bar{\epsilon}_{22} \\
\bar{Y}_{11} & \bar{\mu}_{12} & \bar{\mu}_{11} & \bar{Y}_{12}
\end{array}\right) .
$$

The characteristic equation for the eigenvalue problem (Equation (9)) is a quartic equation equivalent to Equation (1), which is valid for a generic bianisotropic medium without an assumption of reciprocity

$$
n^{4}-\operatorname{tr}(\hat{N}) n^{3}-\xi n^{2}-\zeta n+\operatorname{det}(\hat{N})=0
$$

where

$$
\begin{gathered}
\xi=\frac{1}{2}\left(\operatorname{tr}\left(\hat{N}^{2}\right)-\operatorname{tr}(\hat{N})^{2}\right) \\
\zeta=\frac{1}{6}\left(2 \operatorname{tr}\left(\hat{N}^{3}\right)-3 \operatorname{tr}\left(\hat{N}^{2}\right) \operatorname{tr}(\hat{N})+\operatorname{tr}(\hat{N})^{3}\right)
\end{gathered}
$$

Consider reciprocal materials. The material parameters are bound by $\hat{\epsilon}^{T}=\hat{\epsilon}, \hat{\mu}^{T}=\hat{\mu}, \hat{X}^{T}=-\hat{Y}$, which is true in the transformed coordinates for the elements of $\widetilde{\epsilon}, \widetilde{\mu}, \widetilde{X}, \widetilde{Y}$ and for the elements of $\bar{m}$ in Equations (8) and (9). In this case $\operatorname{tr}(\hat{N})=\operatorname{tr}\left(\hat{N}^{3}\right)=0$, which turns Equation (10) into

$$
n^{4}-\frac{1}{2} \operatorname{tr}\left(\hat{N}^{2}\right) n^{2}+\operatorname{det}(\hat{N})=0
$$

The roots of Equation (11) are

$$
n^{2}=\frac{1}{4}\left\{\operatorname{tr}\left(\hat{N}^{2}\right) \pm \sqrt{\left(\operatorname{tr}\left(\hat{N}^{2}\right)\right)^{2}-16 \operatorname{det} \hat{N}}\right\}
$$

This is an explicit expression for the refraction indices of waves in arbitrary reciprocal materials, and this confirms our previous conclusion from Reference [11], that isofrequency k-surfaces have reflection symmetry in reciprocal materials.

\section{Results}

Let us turn to the asymptotic behavior at high $\mathrm{k}$. If one of the eigenvalues of the index of refraction operator $\hat{N}$ becomes infinite, then $\operatorname{det} \hat{N}=n_{1} n_{2} n_{3} n_{4} \rightarrow \infty$ also diverges, since it is the product of all the eigenvalues $n_{i}$. If the elements of the matrix $\hat{M}$ are finite, then, according to the expression for matrix $\bar{m}$ (Equation (8)), whose elements compose $\hat{N}$ (Equation (9)), such divergence is only possible for waves propagating in directions $\hat{k}$ such that

$$
\Delta_{\infty}=\operatorname{det}\left(\begin{array}{ll}
\widetilde{\epsilon}_{33} & \widetilde{X}_{33} \\
\widetilde{Y}_{33} & \widetilde{\mu}_{33}
\end{array}\right)=0 .
$$

Considering Equation (6) and that $q_{3}=\hat{k}$, Equation (13) can be rewritten as an equation of a quartic conical surface

$$
h(\boldsymbol{k})=0,
$$

where $h(\boldsymbol{k})=\left(\boldsymbol{k}^{T} \hat{\boldsymbol{\epsilon}} \boldsymbol{k}\right)\left(\boldsymbol{k}^{T} \hat{\mu} \boldsymbol{k}\right)-\left(\boldsymbol{k}^{T} \hat{X} \boldsymbol{k}\right)\left(\boldsymbol{k}^{T} \hat{Y} \boldsymbol{k}\right)$ for a generic bianisotropic medium without an assumption of reciprocity.

Direct comparison shows that function $h(\boldsymbol{k})$ of Equation (14) is identical to Equation (2). Note that functions $a(\boldsymbol{k})=\left(\boldsymbol{k}^{T} \hat{a} \boldsymbol{k}\right)=\sum_{i j} a_{i j} k_{i} k_{j}$, where $\hat{a}=\hat{\epsilon}, \hat{\mu}, \hat{X}, \hat{Y}$, are quadratic forms on k-space. 
If $\Delta_{\infty} \rightarrow 0$, then the longitudinal components of fields $E_{z}$ and $H_{z}$ are much greater than the transverse components, so $\boldsymbol{E}=E_{z} \hat{\boldsymbol{k}}, \boldsymbol{H}=H_{z} \hat{\boldsymbol{k}}$. Consequently, Maxwell's equations can be written as

$$
\begin{aligned}
& \boldsymbol{k} \cdot \boldsymbol{D}=\left(\boldsymbol{k}^{T} \hat{\boldsymbol{\epsilon}} \boldsymbol{k}\right) E_{z}+\left(\boldsymbol{k}^{T} \hat{X} \boldsymbol{k}\right) H_{z}=0, \\
& \boldsymbol{k} \cdot \boldsymbol{B}=\left(\boldsymbol{k}^{T} \hat{\mu} \boldsymbol{k}\right) H_{z}+\left(\boldsymbol{k}^{T} \hat{Y} \boldsymbol{k}\right) E_{z}=0 .
\end{aligned}
$$

Equations (15) and (16) have a nonzero solution for the longitudinal fields $\left(E_{z}, H_{z}\right)$ only if Equation (14) is met. Note that from $k \times E=k_{0} \boldsymbol{B}$ and $\boldsymbol{k} \times \boldsymbol{H}=-k_{0} \boldsymbol{D}$, it follows that for HKPs $\boldsymbol{D}=\boldsymbol{B}=0$, since HKP fields are purely longitudinal $\boldsymbol{E}=E_{z} \hat{\boldsymbol{k}}, \boldsymbol{H}=H_{z} \hat{\boldsymbol{k}}$.

An important characteristic of an HKP is its longitudinal impedance, which we introduce using Equations (15) and (16):

$$
Z_{l}=\frac{E_{z}}{H_{z}}=-\frac{\left(k^{T} \hat{X} k\right)}{\left(k^{T} \hat{\epsilon} k\right)}=-\frac{\left(k^{T} \hat{\mu} k\right)}{\left(k^{T} \hat{Y} k\right)}
$$

For reciprocal materials, $\left(\boldsymbol{k}^{T} \hat{Y} \boldsymbol{k}\right)=-\left(\boldsymbol{k}^{T} \hat{X} \boldsymbol{k}\right)$ and Equation (14) breaks into two solution branches:

$$
\sqrt{\left(\boldsymbol{k}^{T} \hat{\epsilon} \boldsymbol{k}\right)\left(\boldsymbol{k}^{T} \hat{\mu} \boldsymbol{k}\right)} \pm i\left(\boldsymbol{k}^{T} \hat{X} \boldsymbol{k}\right)=0
$$

corresponding to impedances

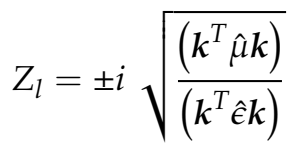

The conventional condition of hyperbolicity requires the principle values of the dielectric permittivity tensor $\hat{\epsilon}$ to be of different signs [2-4]. Indeed, for such materials, $h(\boldsymbol{k})=\left(\boldsymbol{k}^{T} \hat{e} \boldsymbol{k}\right)$ can only be zero if principle values of $\hat{\epsilon}$ have different signs. According to Equations (17) and (19), the longitudinal impedance for the HKP in these materials is $Z_{l}^{-1}=0$. Similarly, for magnetic hyperbolic materials [12], the function from Equation (17) is $h(\boldsymbol{k})=\epsilon\left(\boldsymbol{k}^{T} \hat{\mu} \boldsymbol{k}\right)$, assuming $\epsilon$ is a scalar, and the tensor $\hat{\mu}$ has to have principle values of different signs. The HKP waves have $Z_{l}=0$ in magnetic hyperbolic materials (see Equations (17) and (19)).

It has been theoretically predicted in Reference [7] that in the absence of magnetoelectric coupling $(\hat{X}=\hat{Y}=\hat{0})$, if both $\hat{\epsilon}$ and $\hat{\mu}$ have principal values of different signs at the same frequency $\omega$, then bihyperboloid isofrequency k-surfaces are possible. As can be seen from Equation (14), if $\hat{X}=\hat{Y}=\hat{0}$, then $h(\boldsymbol{k})=\left(\boldsymbol{k}^{T} \hat{\boldsymbol{\epsilon}} \boldsymbol{k}\right)\left(\boldsymbol{k}^{T} \hat{\mu} \boldsymbol{k}\right)$ and, indeed, two hyperboloids in the k-surface can form corresponding to electric $Z_{l}^{-1}=0$ branch with $\left(\boldsymbol{k}^{T} \hat{\boldsymbol{e}} \boldsymbol{k}\right)=0$ and magnetic branch $Z_{l}=0$ with $\left(\boldsymbol{k}^{T} \hat{\mu} \boldsymbol{k}\right)=0$ (cf. the discussion of Figure 3).

Let us consider anisotropic magnetoelectric coupling and study the drastic changes to the topology of the isofrequency surfaces it leads to. We first study materials which are nonhyperbolic in the absence of magnetoelectric coupling, i.e., the principal values of their $\hat{\epsilon}$ and $\hat{\mu}$ tensors have the same signs.

Consider a material with $\hat{\epsilon}=\epsilon \hat{1}, \hat{\mu}=\mu \hat{1}$, and $\hat{Y}=-\hat{X}^{T}=\operatorname{diag}\left(i \kappa_{1}, i \kappa_{1}, i \kappa_{2}\right)$. Equation (14) then turns into $\left(q_{\rho}^{2} \kappa_{1}+q_{z}^{2} \kappa_{2}\right)^{2}=\epsilon \mu$, which shows that HKPs propagate if $\left(\kappa_{1}^{2}-\epsilon \mu\right)$ and $\left(\kappa_{2}^{2}-\epsilon \mu\right)$ have opposite signs. Note that $\kappa=\kappa_{1}=\kappa_{2}= \pm \sqrt{\epsilon \mu}$ does not lead to the formation of HKPs, since in this case a 0/0 indeterminacy forms in Equation (9), which resolves as 0, since for an isotropic chiral material $n=\sqrt{\epsilon \mu} \pm \kappa$ [13]. The formation of a hyperbolic material here is due to the anisotropic chirality tensor and is shown in Figure 2a. 
(a)

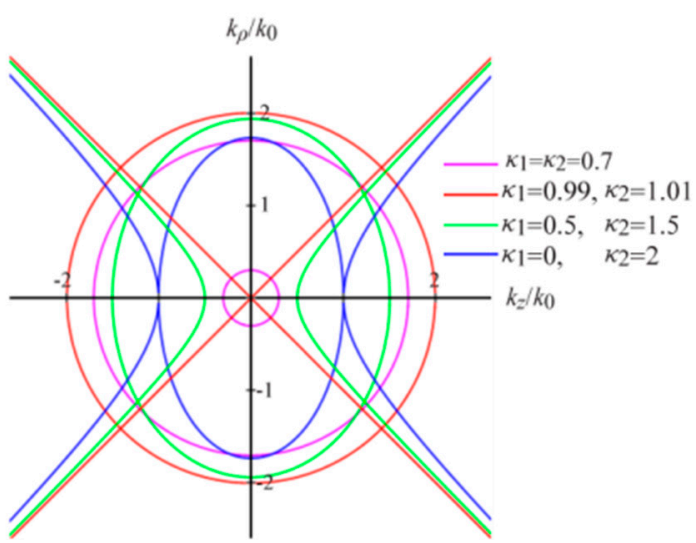

(c)
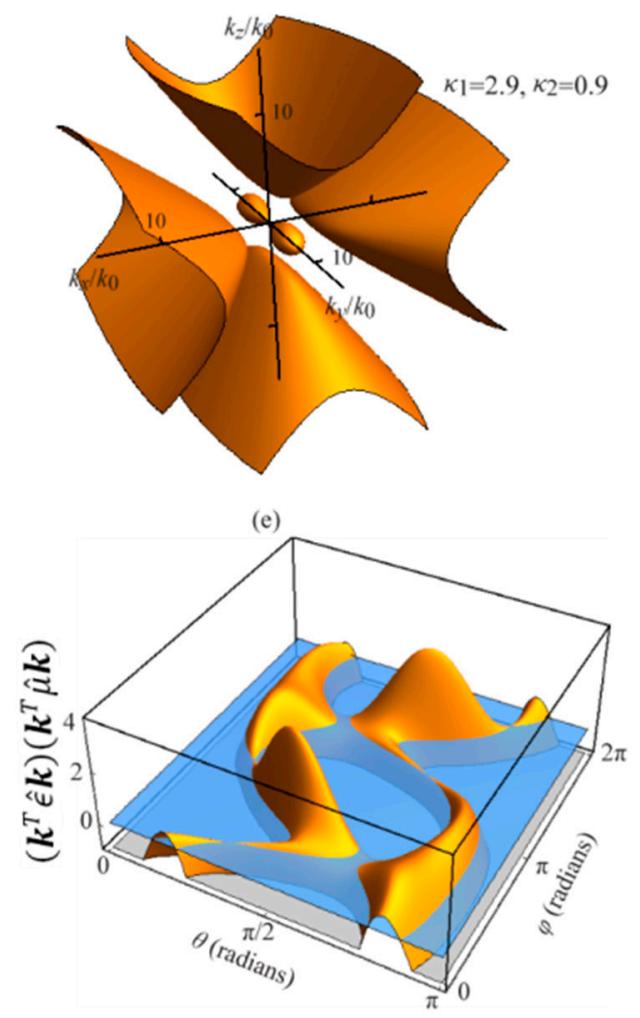

(b)

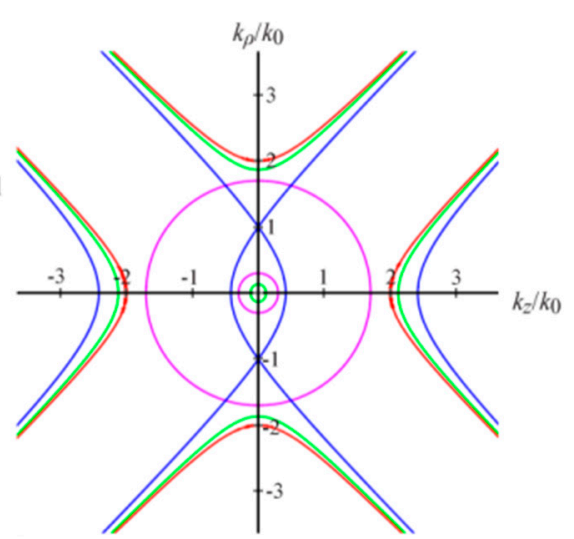

(d)

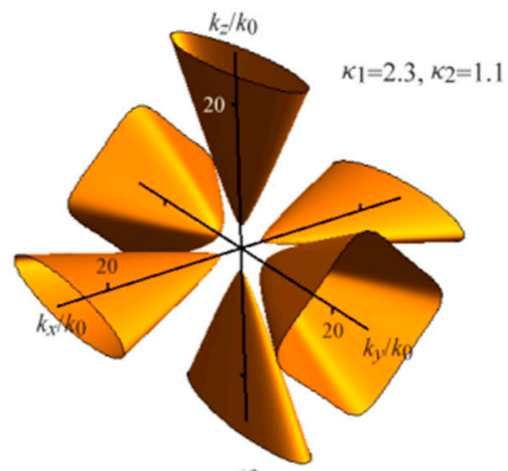

(f)

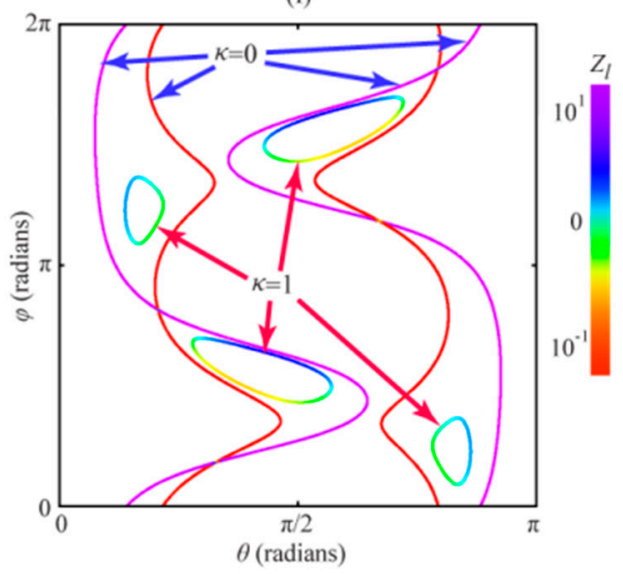

Figure 2. (a-d) Chirality-inducing hyperbolicity in nonhyperbolic phases (material parameters detailed in text). (e,f) Chirality-induced phase modifications in anisotropic materials. (e) Plot of the function $\left(\boldsymbol{k}^{T} \hat{\epsilon} \boldsymbol{k}\right)\left(\boldsymbol{k}^{T} \hat{\mu} \boldsymbol{k}\right)$ for the $\hat{\epsilon}, \hat{\mu}$ matrices used in (f) and Figure 3.

If reciprocity is broken $\hat{X}^{T} \neq-\hat{Y}$, then $h(\boldsymbol{k})=\epsilon \mu-\left(\boldsymbol{k}^{T} \hat{X} \boldsymbol{k}\right)\left(\boldsymbol{k}^{T} \hat{Y} \boldsymbol{k}\right)$ and a bihyperboloidal k-surface may form. In Figure $2 b$ we plot $k$-surfaces for a material with $\hat{\epsilon}=\epsilon \hat{1}, \hat{\mu}=\mu \hat{1}, \hat{X}=-i \operatorname{diag}\left(\kappa_{1}, \kappa_{1}, \kappa_{2}\right)$, and $\hat{Y}=i \operatorname{diag}\left(\kappa_{2}, \kappa_{2}, \kappa_{1}\right)$ for the same values of $\kappa_{1}$ and $\kappa_{2}$ as in Figure $2 \mathrm{a}$ and demonstrate the bihyperbolic dispersion.

In Figure 2c,d we demonstrate that a material with anisotropic $\hat{\epsilon}=\operatorname{diag}(1,1,5), \hat{\mu}=\operatorname{diag}(5,1,1)$, which is nonhyperbolic without magnetoelectric coupling, forms bihyperbolic and trihyperbolic phases if anisotropic chirality $\hat{Y}=-\hat{X}^{T}=\operatorname{diag}\left(i \kappa_{1}, i \kappa_{2}, i \kappa_{1}\right)$ is added with $\kappa_{1}=2.9$ and $\kappa_{2}=0.9$ for Figure $2 \mathrm{c}$ and $\kappa_{1}=2.3$ and $\kappa_{2}=1.1$ for Figure $2 \mathrm{~d}$. 
(a)
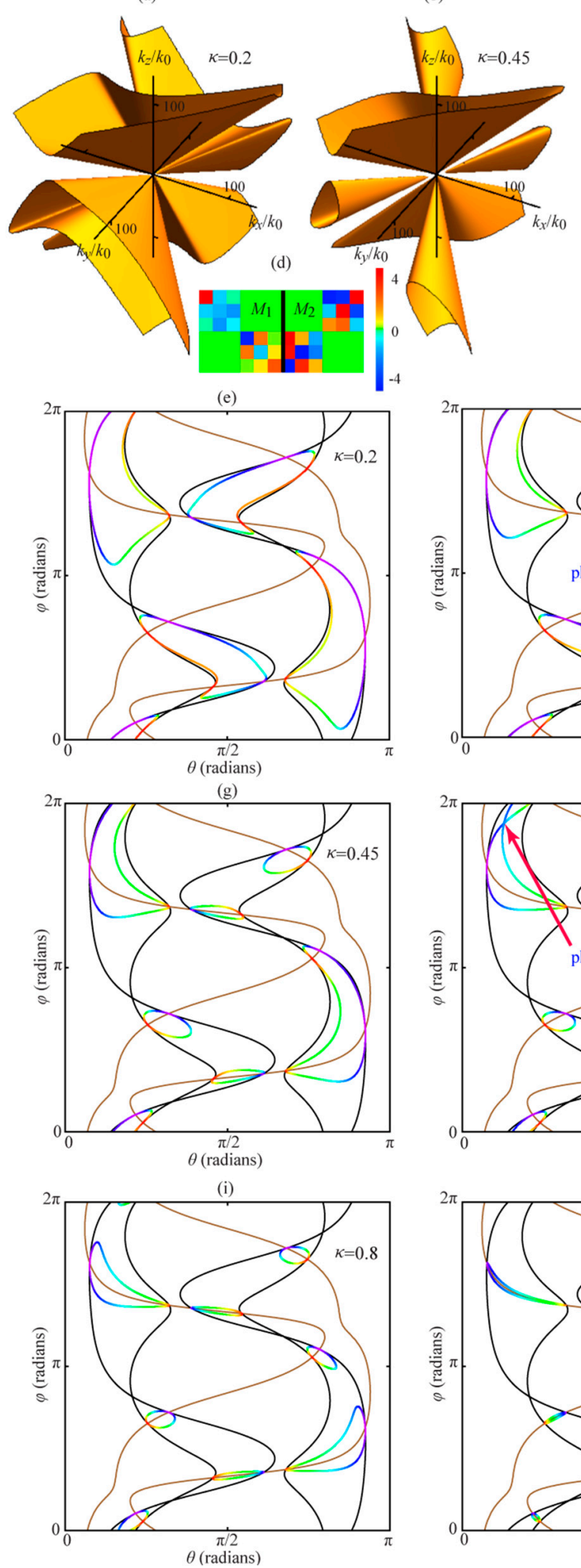

(c)

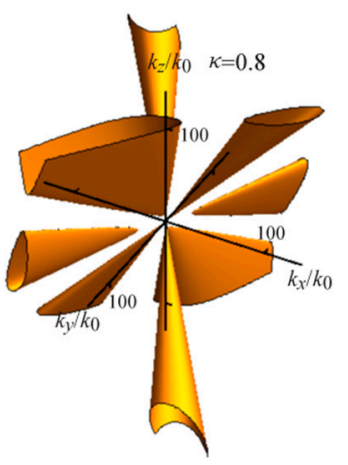

(f)
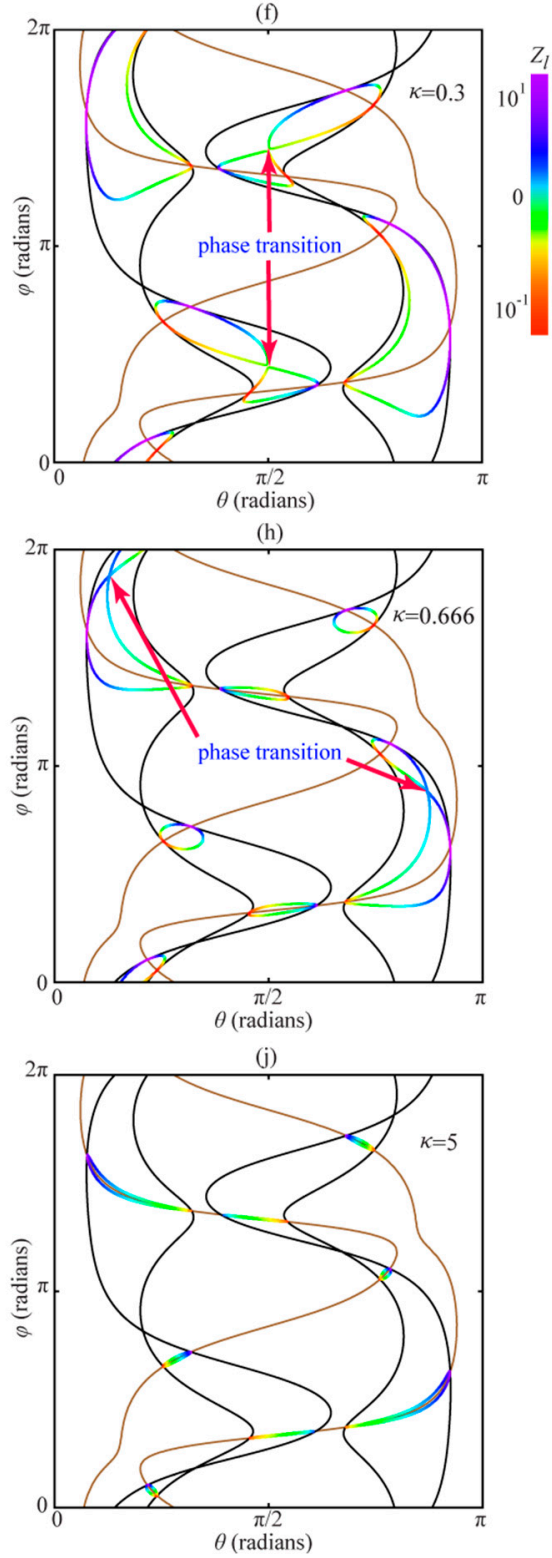

Figure 3. Topological phase transitions $(\mathbf{f}, \mathbf{h})$ between bihyperbolic $(\mathbf{a}, \mathbf{e})$, trihyperbolic, $(\mathbf{b}, \mathbf{g})$ and tetrahyperbolic $(\mathbf{c}, \mathbf{i})$ phases with material parameters matrix $\hat{M}=\hat{M}_{1}+i \kappa \hat{M}_{2}$ as $\kappa$ is changed. Panel (j) shows the limit of high $\kappa$. Matrices $M_{1}, M_{2}$ are plotted in (d). 
Now let us turn to materials that are hyperbolic in the absence of magnetoelectric coupling and consider how magnetoelectric coupling changes their topologies and HKPs. Below we consider a numerical example of matrix $M_{1}=\left[\begin{array}{ll}\hat{\epsilon} & 0 \\ 0 & \hat{\mu}\end{array}\right]$ shown in the inset Figure 3d (to the left of the black line). This matrix $M_{1}$ describes an anisotropic material with the dispersion transitional between hyperbolic and bihyperbolic. The HKP propagation directions for $M_{1}$ are illustrated in Figure 2e,f. In Figure 2e we plot the function $\left(\boldsymbol{k}^{T} \hat{\boldsymbol{k}} \boldsymbol{k}\right)\left(\boldsymbol{k}^{T} \hat{\mu} \boldsymbol{k}\right)$ (yellow), whose intersection with zero (blue) corresponds to the HKP propagation direction. These directions are also plotted in Figure $2 \mathrm{f}$ for the electric branch $Z_{l}^{-1}=0$, with $\left(\boldsymbol{k}^{T} \hat{\boldsymbol{k}} \boldsymbol{k}\right)=0$ (purple) and magnetic branch $Z_{l}=0$ with $\left(\boldsymbol{k}^{T} \hat{\mu} \boldsymbol{k}\right)=0$ (red), which corresponds to the color scale for $Z_{l}$ shown to the right. Let us turn to a material characterized by matrix $\hat{M}=\hat{M}_{1}+i \kappa \hat{M}_{2}$, where $M_{2}=\left[\begin{array}{cc}\hat{0} & \hat{1} \\ -\hat{1} & \hat{0}\end{array}\right]$ is responsible for magnetoelectric coupling. The change in the HKP propagation directions with increasing $\kappa$ from zero corresponds to raising the level of the blue plane in Figure 2e to level $\kappa^{2}$, which confines the HKP states to the positive region $\left(\boldsymbol{k}^{T} \hat{\boldsymbol{\epsilon}} \boldsymbol{k}\right)\left(\boldsymbol{k}^{T} \hat{\mu} \boldsymbol{k}\right)$. The HKP propagation directions for $\kappa=1$ are shown in Figure $2 \mathrm{f}$ with four disconnected curves, which corresponds to bihyperbolic dispersion. These connectivity curves are color-coded corresponding to $Z_{l}$ of the HKP states.

Let us consider a material described by the matrix $\hat{M}=\hat{M}_{1}+i \kappa \hat{M}_{2}$ with the individual matrices $M_{1}, M_{2}$ plotted on Figure 3d. Changing parameter $\kappa$ leads to topological transitions between bihyperbolic (Figure 3a,e), trihyperbolic (Figure 3b,g), and tetrahyperbolic (Figure 3c,i) phases. The progression of the phase changes is demonstrated in Figure $3 \mathrm{e}-\mathrm{j}$. In these panels the HKP states for $\kappa=0$ satisfying $\left(\boldsymbol{k}^{T} \hat{\boldsymbol{c}} \boldsymbol{k}\right)\left(\boldsymbol{k}^{T} \hat{\mu} \boldsymbol{k}\right)=0$ are outlined in black, which are the same curves as in Figure 2f. The directions for which $\kappa=0$ and $\left(\boldsymbol{k}^{T} \hat{X} \boldsymbol{k}\right)=0$ (brown) satisfy Equations (14) and (18) for all $\kappa$ and serve as the framework for the topological transitions. These intersection points in Figure $3 \mathrm{e}-\mathrm{j}$ have invariant impedances $Z_{l}^{-1}=0$ for the electric branch with $\left(\boldsymbol{k}^{T} \hat{\epsilon} \boldsymbol{k}\right)=0$ and $Z_{l}=0$ for the magnetic branch.

The HKP states for the $\kappa$ noted in panels (e)-(j) are color-coded in accordance with their $Z_{l}$ as indicated to the right of Figure 3f. The HKP for nonzero $\kappa$ are positioned in the $\left(\boldsymbol{k}^{T} \hat{\boldsymbol{e}} \boldsymbol{k}\right)\left(\boldsymbol{k}^{T} \hat{\mu} \boldsymbol{k}\right)>0$ regions between the $\kappa=0$ lines, in accordance with Equations (14) and (18), and touch these lines at the intersections of $\kappa=0$ and $\left(\boldsymbol{k}^{T} \hat{X} \boldsymbol{k}\right)=0$. The HKP states group into several disconnected curves, the number of which characterizes the topology of the phase. Note that each curve is split into two parts by the $\left(\boldsymbol{k}^{T} \hat{X} \boldsymbol{k}\right)=0$ curves at their intersections with $\kappa=0$. The resulting two subcurves of HKP states correspond to two different branches of Equation (18).

The addition of a small $\kappa$ leads to a transition to the bihyperbolic phase (four disconnected curves) as shown in Figure 3e. At $\kappa=0.3$ (Figure 3f), a phase transition occurs from the bihyperbolic to the trihyperbolic phase (six curves) in the k-space direction marked by the red arrows. In this direction the group velocity, which is normal to the $\mathrm{k}$-surface, becomes zero

$$
v_{g}=\nabla_{k} \omega \propto \nabla_{k} f\left(\boldsymbol{k}, k_{0}\right)=\nabla_{k} h(\boldsymbol{k})=0
$$

In general, we identify the condition (Equation (20) with topological phase transitions. The topological phase transition of the isofrequency k-surfaces were first discussed in Reference [14] for the monohyperbolic phase. The transition from the trihyperbolic (Figure 3g) to the tetrahyperbolic phase (eight curves in Figure 3i) occurs at $\kappa=0.666$ (Figure $3 \mathrm{~h}$ ). The k-space direction in which the transition occurs satisfies Equation (20) and is marked by the red arrows. For a large $\kappa$, the HKP states closely follow the $\left(k^{T} \hat{X} k\right)=0$ curves between their intersections with $\kappa=0$. Therefore, in the general case of arbitrary matrices $\hat{M}_{1}$ and $\hat{M}_{2}$, the number of disconnected sections of $\left(\boldsymbol{k}^{T} \hat{X} \boldsymbol{k}\right)=0$ between their intersections with $\kappa=0$ can serve as the prediction of the topological phase of the isofrequency surfaces for a large $\kappa$. 


\section{Conclusions}

To conclude, let us ask the following question-what is the limit on the number of hyperboloids in isofrequency surfaces of bianisotropic materials? To classify the quartic k-surfaces in the $k \gg k_{0}$ limit topologically, it is convenient to use the projective plane P2. As was noted in relation to bianisotropic materials [10], the classification follows from the following theorem [7]—a smooth-projective real quartic curve consists topologically of (i) one oval (hyperbolic medium), (ii) two non-nested ovals (bihyperbolic medium), (iii) two nested ovals, (iv) three ovals (trihyperbolic medium), (v) four ovals (tetrahyperbolic medium), and (vi) an empty set (nonhyperbolic medium).

Therefore, in the present manuscript we complete the topological classification of bianisotropic optical materials.

Author Contributions: Conceptualization, M.D.; methodology, M.D.; investigation, R.W., M.L., T.M., and M.D.; resources, M.D.; writing—original draft preparation, M.D.; writing—review and editing, R.W., M.L., and T.M.; supervision, M.D.; project administration, M.D.; funding acquisition, M.D. All authors have read and agreed to the published version of the manuscript.

Funding: Summer Research Session and College Office of Undergraduate Research (COUR) Grants from the College of Science and Mathematics at Georgia Southern University.

Conflicts of Interest: The authors declare no conflict of interest.

\section{References}

1. Tolstoy, A.N. The Hyperboloid of Engineer Garin; State Publisher: Moscow, USSR, 1929; Volume 10.

2. Lindell, I.V.; Tretyakov, S.A.; Nikoskinen, K.I.; Ilvonen, S. BW media - Media with negative parameters, capable of supporting backward waves. Microw. Opt. Technol. Lett. 2001, 31, 129-133. [CrossRef]

3. Smith, D.R.; Kolinko, P.; Schurig, D. Negative refraction in indefinite media. JOSA B 2004, 21, 1032. [CrossRef]

4. Podolskiy, V.A.; Narimanov, E.E. Strongly anisotropic waveguide as a nonmagnetic left-handed system. Phys. Rev. B 2005, 71. [CrossRef]

5. Tuz, V.R.; Fedorin, I.V.; Fesenko, V.I. Bi-hyperbolic isofrequency surface in a magnetic-semiconductor superlattice. Opt. Lett. 2017, 42, 4561. [CrossRef] [PubMed]

6. Noginov, M.A.; Podolskiy, V.A. (Eds.) Tutorials in Metamaterials; CRC Press: Boca Raton, FL, USA, 2011.

7. Zeuthen, H.G. Sur les différentes formes des courbes planes du quatrième ordre. Math. Ann. 1873, 7, 408-432.

8. Gao, W.; Lawrence, M.; Yang, B.; Liu, F.; Fang, F.; Béri, B.; Li, J.; Zhang, S. Topological Photonic Phase in Chiral Hyperbolic Metamaterials. Phys. Rev. Lett. 2015, 114. [CrossRef] [PubMed]

9. Mills, D.L.; Burstein, E. Polaritons: The electromagnetic modes of media. Rep. Prog. Phys. 1974, 37, 817. [CrossRef]

10. Mulkey, T.; Dillies, J.; Durach, M. The Inverse Problem of Quartic Photonics. Opt. Lett. 2018, 43, 1226-1229. [CrossRef] [PubMed]

11. LaBalle, M.; Durach, M. Additional waves and additional boundary conditions in local quartic metamaterials. OSA Contin. 2019, 2, 17. [CrossRef]

12. Kruk, S.S.; Zi, J.W.; Pshenay-Severin, E.; O'Brien, K.; Neshev, D.N.; Kivshar, Y.S.; Zhang, X. Magnetic hyperbolic optical metamaterials. Nat. Commun. 2016, 7. [CrossRef] [PubMed]

13. Zhang, S.; Park, Y.S.; Li, J.; Lu, X.; Zhang, W.; Zhang, X. Negative refractive index in chiral metamaterials. Phys. Rev. Lett. 2009, 102. [CrossRef] [PubMed]

14. Krishnamoorthy, N.H.; Jacob, Z.; Narimanov, E.; Kretzschmar, I.; Menon, V.M. Topological transitions in metamaterials. Science 2012, 336, 205-209. [CrossRef] [PubMed] 